\title{
Graphing within-subjects confidence intervals using SPSS and S-Plus
}

\author{
DANIEL B. WRIGHT \\ University of Sussex, Brighton, England
}

\begin{abstract}
Within-subjects confidence intervals are often appropriate to report and to display. Loftus and Masson (1994) have reported methods to calculate these, and their use is becoming common. In the present article, procedures for calculating within-subjects confidence intervals in SPSS and S-Plus are presented (an R version is on the accompanying Web site). The procedure in S-Plus allows the user to report the bias corrected and adjusted bootstrap confidence intervals as well as the standard confidence intervals based on traditional methods. The presented code can be easily altered to fit the individual user's needs.
\end{abstract}

The American Psychological Association's task force on statistical inference (Wilkinson et al., 1999) and many other journals' and societies' guidelines (e.g., Thompson, 1996; Wright, 2003) encourage authors to produce confidence intervals. In much psychology research, the main interest is comparing the scores of participants who are in several conditions-so-called within-subjects designs. Most of the standard statistical packages, however, only produce confidence intervals that are appropriate for between-subjects comparisons. Code is presented here to produce within-subjects confidence intervals and their accompanying graphs with SPSS and S-Plus.

Loftus and Masson (1994; Masson \& Loftus, 2003; see also Estes, 1997; Wright, 1997) described how withinsubjects confidence intervals can be calculated. Their approach has become popular - their original article being cited over 500 times (Web of Science, wok.mimas.ac.uk, accessed April 22, 2007). The present article uses their procedure. Loftus and Masson discussed in detail how these intervals should be used. The most important point to note is that these intervals do not show the precision in the estimate of the means, but instead show precision in patterns among the means, which (according to Loftus and Masson) is what psychologists are most often interested in. They argue that these graphs help to communicate patterns in the data that are not as easily communicated through statistical tests and traditional confidence interval graphs.

Consider the hypothetical data in Table 1 for 10 children in four different within-subjects conditions. Following Loftus and Masson (1994), the means for each child are calculated and then the grand mean is calculated. Here, it is 4.35. An adjustment value for each individual is calculated that is the grand mean minus the individual's mean. Thus, for the first child, this is $4.35-2.50=1.85$. This value is then added to every value for this individual. These new values can then be used to construct confi- dence intervals, and these can be graphed. They are the data with the individual variation removed; however, they still maintain the differences among the conditions (Loftus \& Masson, 1994).

Suppose the data in Table 1 are in an SPSS data file (.sav) where the variables - called cond1 to cond 4 - are contiguous in a data file called $\mathrm{c}: \backslash$ temp $\backslash \mathrm{child}$. sav. The code given in the Appendix (also available from www .sussex.ac.uk/Users/danw/WSCI/wscihome.htm and the Psychonomic Society archive) defines an SPSS macro. If included, the command (where WSCI stands for "withinsubjects confidence interval")

$$
\begin{gathered}
\text { !WSCI fpath="c: } \backslash \text { temp \child.sav" } \\
\text { k=4 ci=95 v=condl to cond4. }
\end{gathered}
$$

will produce an error bar diagram based on the $95 \%$ within-subjects confidence intervals. The first part of the command tells the computer where the data file is located. The variable $k$ is the number of conditions. The variable $c i$ allows the user to adjust the level of confidence. Values should range from 0 to 100 , where 95 equates with $95 \%$ confidence intervals. Values outside this will result in an error. Values less than 1 -most likely due to entering .90, thinking this was 90 -will result in very small intervals. The narrowness of the intervals should make this error obvious to all users. The variables are then input in the format of the first variable to include the word $\mathrm{TO}$, then the final variable. All these parameters must be entered for the macro to work (i.e., there are no default values for these).

The adjusted values are saved in a file called $\mathrm{c}: \backslash$ temp $\backslash$ adjusted.sav. Thus, the code assumes there is a $\mathrm{c}: \backslash$ temp directory on the computer. If not, the code can be easily changed. The means and confidence intervals are then printed. It is assumed that most researchers will want to use these values to tailor the graph so that it best suits their individual needs. When the number of partici-

D. B.Wright, danw@sussex.ac.uk 
Table 1

Data for 10 Children in Four Different Conditions

\begin{tabular}{|c|c|c|c|c|c|c|c|c|c|c|c|}
\hline & \multicolumn{10}{|c|}{ Child Number } & \multirow{2}{*}{$\begin{array}{c}\text { Condition } \\
\text { Mean }\end{array}$} \\
\hline & 1 & 2 & 3 & 4 & 5 & 6 & 7 & 8 & 9 & 10 & \\
\hline Condition 1 & 2 & 5 & 6 & 1 & 1 & 6 & 6 & 5 & 2 & 1 & 3.5 \\
\hline Condition 2 & 2 & 5 & 5 & 7 & 0 & 8 & 2 & 3 & 1 & 1 & 3.4 \\
\hline Condition 3 & 4 & 6 & 9 & 5 & 2 & 9 & 6 & 6 & 1 & 0 & 4.8 \\
\hline Condition 4 & 2 & 6 & 10 & 8 & 5 & 11 & 5 & 8 & 0 & 2 & 5.7 \\
\hline Child mean & 2.50 & 5.50 & 7.50 & 5.25 & 2.00 & 8.50 & 4.75 & 5.50 & 1.00 & 1.00 & \multirow{2}{*}{$\mathrm{GM}=4.35$} \\
\hline Adj. value & 1.85 & -1.15 & -3.15 & -0.90 & 2.35 & -4.15 & -0.40 & -1.15 & 3.35 & 3.35 & \\
\hline
\end{tabular}

Note-GM, grand mean. Adj. value is the adjustment value, which is the grand mean minus the participant's mean.

pants is small, it is often informative to graph separate lines for each participant. Within SPSS, this can be done with line graph.

The function was also implemented in S-Plus (and an R version is on the Web site). For most confidence intervals that psychologists construct, it is assumed that the data are normally distributed. Bootstrapping procedures are becoming popular for estimating confidence intervals when this assumption is suspect. For this function, the user has the option of producing bias corrected and adjusted (BCa) bootstrap confidence limits. The $\mathrm{BCa}$ procedure is one of several methods for constructing bootstrap intervals and has several good properties (see DiCiccio \& Efron, 1996, for details). It is assumed that an S-Plus file with these data have been attached. The command

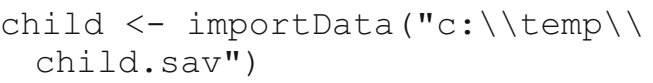

can be used to import the data from SPSS. The following code calls the WSCI function:

$$
\text { wsci ( } \operatorname{child}[, 1: 4], \operatorname{meth}=1)
$$

The within-subjects variables are in the first four columns. Any columns can be specified in the square brackets after the comma; they do not need to be contiguous. The code

$$
\text { wsci ( child }[, \mathrm{c}(1,3,5,7)])
$$

will perform the analysis on the first, third, fifth, and seventh variables. The default method for constructing the confidence intervals uses the traditional $\pm t S E$ method. This is meth $=0$. BCa is used if met $h=1$. The $95 \%$ confidence interval is used here. Use the following to construct the same graph for the $90 \% \mathrm{BCa}$ within-subjects confidence interval:

$$
\text { wsci ( } \operatorname{child}[, 1: 4] \text {, meth=1, interval=90) }
$$

As with the SPSS procedure, the interval should run from 0 to 100 and so be in percentages. The output from the function is the means and confidence limits in both tabular and graphical forms. It is assumed that some people will use these numbers to create graphs that are specifically tailored for their individual needs.

There are a few differences between the SPSS macro and the S-Plus function. The main difference is that the SPSS macro uses the error bar graph. This was not used in S-Plus because in order to allow BCa confidence limits, it was necessary to input the actual values for the means and the limits of the interval, and these were input into a high-low graph (a type of graph often used with time series analysis in economics).

As the code is presented, users are welcomed to extend it to suit their individual needs. For example, there are several macros for bootstrap procedures in SPSS that can be downloaded and incorporated into the SPSS macro (e.g., David Hitchin's BCNON macro available at spsstools.net/ Syntax/Bootstrap/bcnon.txt). If the method based on bootstrap estimates was to be used, the SPSS HILO graph would also need to be used. The S-Plus function can be extended in similar ways. Because publication-quality graphics usually require specific tailoring, only basic graphs are produced by the functions. The graphs created can be edited in graph editors of SPSS and S-Plus, respectively (as has been done in the Appendix). Only the simplest within-subjects design is used. More complex designs, including contrasts, are discussed in Masson and Loftus (2003). Updated information is available on the author's Web page: www.sussex.ac.uk/Users/danw/.

\section{AUTHOR NOTE}

Address correspondence to D. B. Wright, Psychology Department, University of Sussex, Brighton BN1 9QH, England (e-mail: danw@ sussex.ac.uk).

\section{REFERENCES}

DiCiccio, T. J., \& EFron, B. (1996). Bootstrap confidence intervals. Statistical Science, 11, 189-228.

EsTES, W. K. (1997). On the communication of information by displays of standard errors and confidence intervals. Psychonomic Bulletin \& Review, 4, 330-341.

Loftus, G. R., \& Masson, M. E. J. (1994). Using confidence intervals in within-subject designs. Psychonomic Bulletin \& Review, 1, 476-490.

Masson, M. E. J., \& Loftus, G. R. (2003). Using confidence intervals for graphically based data interpretation. Canadian Journal of Experimental Psychology, 57, 203-220.

THOMPSON, B. (1996). AERA editorial policies regarding statistical significance testing: Three suggested reforms. Educational Researcher, 25, 26-30.

Wilkinson, L., \& the Task Force on Statistical Inference (1999). Statistical methods in psychology journals: Guidelines and explanations. American Psychologist, 54, 594-604.

Wright, D. B. (1997). Understanding statistics: An introduction for the social sciences. London: Sage.

Wright, D. B. (2003). Making friends with your data: Improving how statistics are conducted and reported. British Journal of Educational Psychology, 73, 123-136. 


\section{APPENDIX}

\section{SPSS Macro}

This macro assumes that there is a $\mathrm{c}: \backslash$ temp directory. fpath is for the file's location. $k$ is for the number of within-subjects variables. $c i$ is for the confidence level ranging from 0 to $100 . v$ is for the list of variables. The variables must be contiguous and referred to with the first variable name TO the last variable name. This is necessary for the VECTOR command.

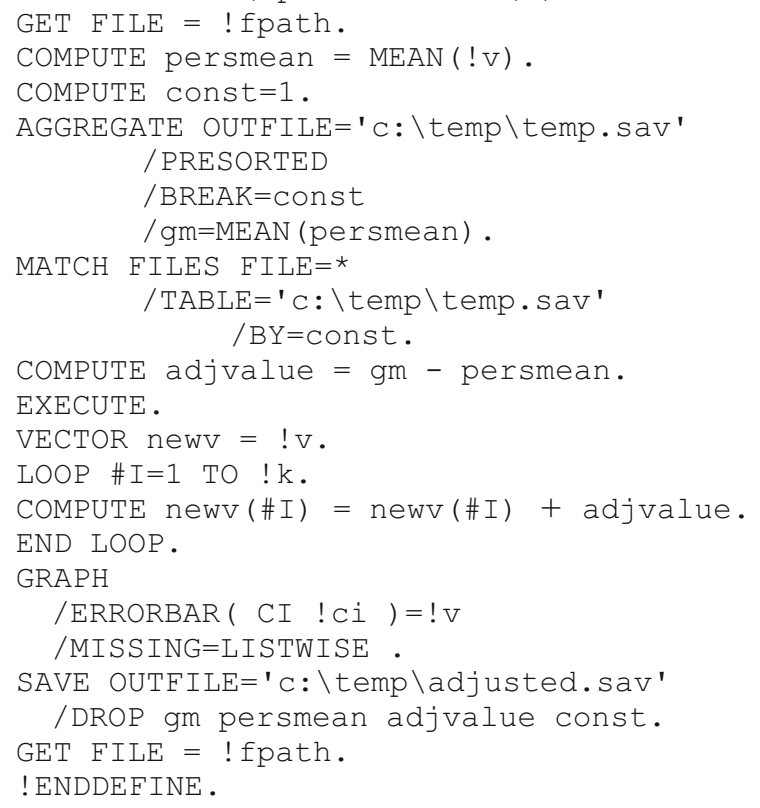

Example:

!WSCI fpath="c: \temp \child.sav" k=4 ci=90 v=condl TO cond4.

\section{S-Plus Function}

mat refers the active data set. If the data set is not active, it should be attached, for example, attach (child). If it is in another format, it should be imported with the importData function, for example, importData ("c: \\temp \\child. sav"), where the $\backslash \backslash$ are used instead of $\backslash$, so that they are interpreted properly. Any variable in the data set can be specified by using the concatenating function, $c$. If all the variables are used, then only the name of the data set is necessary. meth refers to whether traditional confidence intervals are used, met $\mathrm{h}=0$, or whether $\mathrm{BCa}$ intervals are used, meth=1. interval refers to confidence level, ranging from 0 to 100 .

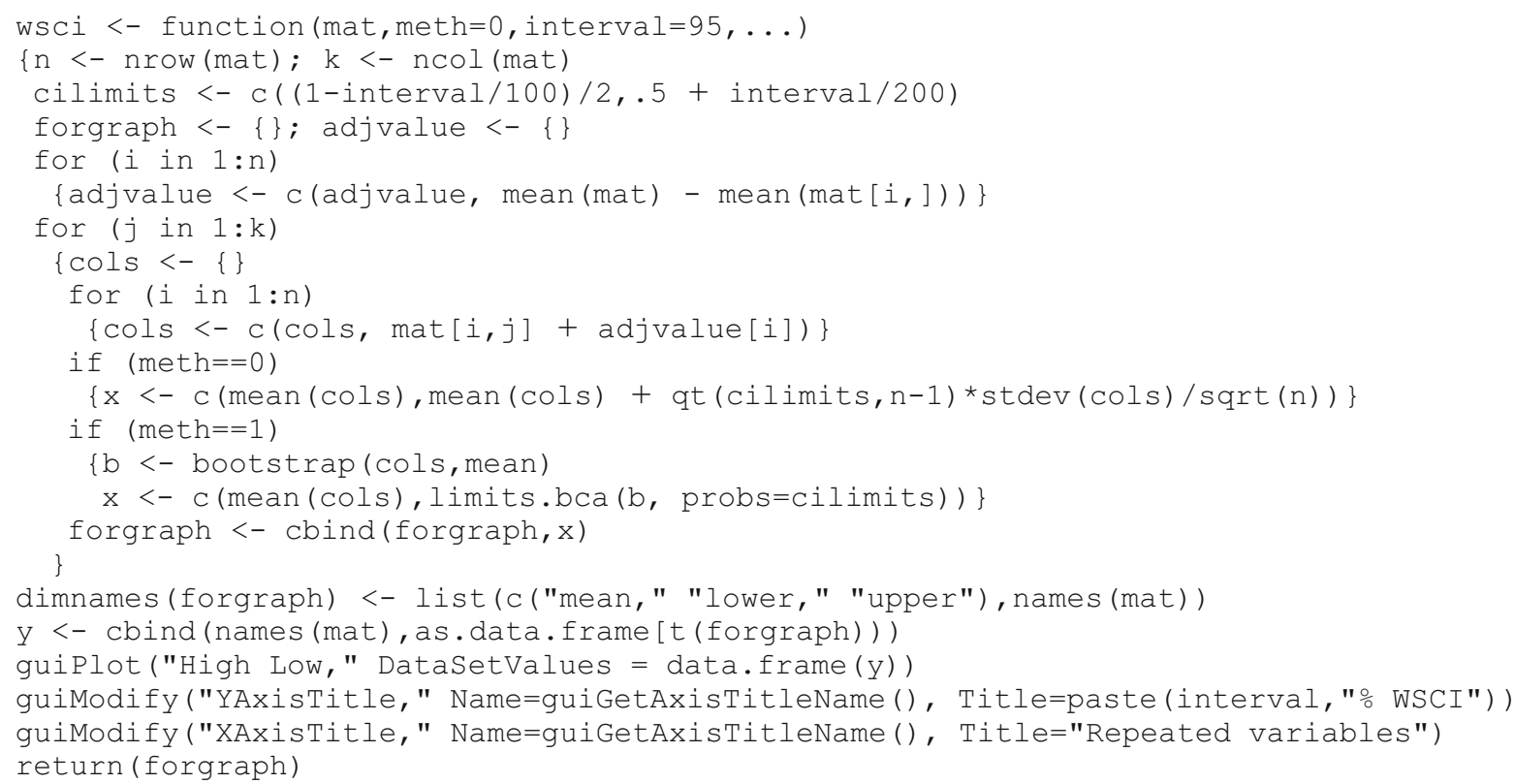


APPENDIX (Continued)

\section{Examples (Shown in Figure A1):}

\# A simple example, 95\% confidence interval

wsci (child [, 1:4])

\# One using BCa intervals and 90\% confidence interval

wsci (child $[, 1: 4]$, meth=1, interval=90)

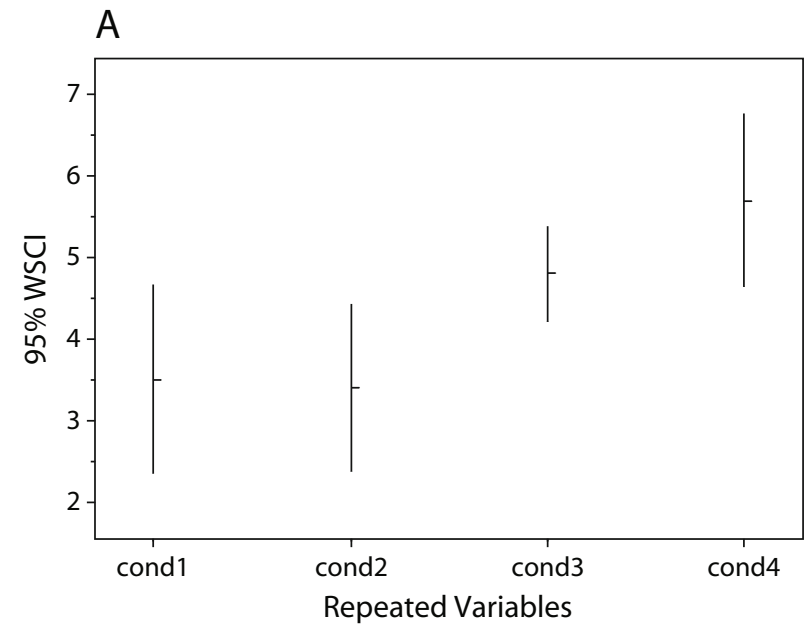

B

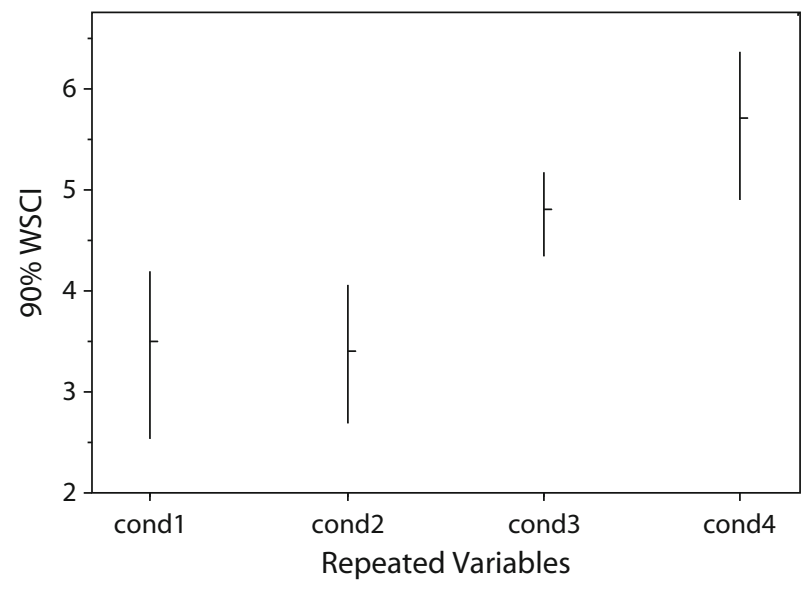

Figure A1. Two within-subjects confidence interval (WSCI) graphs made with the S-Plus function. Figure A1A is the $95 \%$ confidence interval made in the traditional way. Figure A1B is the $\mathbf{9 0 \%}$ confidence interval made using BCa intervals.

An $\mathrm{R}$ version, with additional features (e.g., it can include graphs with both between- and within-subjects confidence intervals), is available on the Web site. It requires functions in the boot and Hmisc libraries, so these should be loaded. The code source(“http://www.sussex.ac.uk//Users//danw//WSCI//wsci.R”) will access it from R.

(Manuscript received May 20, 2005;

revision accepted for publication October 10, 2005.) 